\title{
Hepatic Artery Infusion: Still an Option?
}

\author{
Lawrence D. Wagman, MD, FACS
}

The Center for Cancer Prevention and Treatment, St Joseph Hospital of Orange, Orange, CA

Time and science have been kind to patients with colorectal cancer metastatic to the liver. The combination of a decrease in surgical mortality, increased operative tools, increase in the number of active chemotherapeutic drugsboth general and targeted-accompanied by an acceptance of multimodality therapies have improved patient outcomes. A few tools have maintained a quiet hum in the treatment cacophony. The focus of the paper by Ammori et al. is one of those tools: the continuous hepatic artery infusion (CHAI) pump. Emerging in the 1980s as an effective method of delivering floxuridine to the liver via the hepatic artery, its success was consistent but simultaneously limited to a few centers and physicians (medical oncologists and surgeons) who had adequate and specific skill in using this treatment modality. There were many challenges to the use of continuous infusion hepatic artery delivery of floxuridine. They included the complexities of managing the idiopathic liver transaminitis with appropriate dose reductions, surgically placing the pump and working through the vagaries of the hepatic arterial anatomy, dealing with the mechanical challenges of accessing the chambers for filling, and removing the drug and replacing with heparinized saline, and a continued surveillance for late biliary sclerosis. Clearly, using the technology in the "adjuvant setting," i.e., following hepatic resection for colorectal metastases, was most problematic. The saving grace in this setting was that the patient did not require an additional surgery for the pump placement; however, concerns of functional liver reserve and recovery created an enhanced anxiety. Despite all the challenges, the committed group of oncologists (medical and surgical) consistently demonstrated in pilot and phase 2 studies, a series of outcomes that exceeded those generated with only the systemic chemotherapy available during the same time

(C) Society of Surgical Oncology 2013

Published Online: 21 May 2013

L. D. Wagman, MD, FACS

e-mail: lawrence.wagman@stjoe.org period. When the limited choice for chemotherapy morphed from 5-fluoruracil and leucovorin-excellent agents with known toxicity and carefully studied therapeutic regimensto oxaliplatin, irinotecan, capecitabine, and subsequently the targeted agents led initially by bevacizumab and cetuximab, that's when things changed. An enthusiasm for the new agents with potential for sequential use and improved response rates emerged. The CHAI with the reasonably toxic floxuridine fell from favor and nearly all medical oncologists began to transition their clinical practice to the new systemic agents. A few strongholds of CHAI remained active but the incorporation of pump based chemotherapy rapidly lost ground. The clinical trials that had been developed during the height of interest in pump therapy suffered poor accrual and failed to definitively answer the question of superiority or even equivalence (noninferiority). During this same timeframe came the battle cry for more aggressive surgical approaches following successful "neoadjuvant" conversion chemotherapy. The selection of neoadjuvant treatments were focused on efficacy measured as response and safety as measured by the morbidity and mortality of the subsequent operations. Those practitioners with experience in CHAI accepted and worked around the hepatocyte damage as measured in the hepatic transaminitis and the measurable and presumed intra- and extrahepatic biliary injury that resulted from the chemotherapy-infused blood bathing the biliary structures. Thoughtful attempts to reduce the injury with dexamethasone were admirable but not adequate to consistently reduce hepatic injury. The "soft" hepatic damage of oxaliplatin (sinusoidal injury), fluoropyrimidine and irinotecan (steatosis), and bevacizumab pales compared with a liver arterially infused 2 weeks on and 2 weeks off with floxuridine. It is almost ironic to consider the current restrictions on timing of hepatic surgery after systemic chemotherapy compared with the post-CHAI liver.

So where does this leave us when faced with colorectal cancer metastatic to the liver? Metastatic disease that is resectable at presentation may or may not be treated with 
neoadjuvant chemotherapy to assess responsiveness. In scenarios that are at high risk for intra- and extrahepatic recurrence, the run in period of chemotherapy allows for declaration of a very aggressive biology-preventing therapeutic resections/ablations without benefit. For borderline resectable disease, an analysis of the limits on resection is needed and can be defined. In this situation, the immediate concern is initiation of systemic chemotherapy with known responsiveness to prevent progression to unresectable. The next is drug delivery at projected therapeutically effective doses. While the use if CHAI chemotherapy is attractive based on its response rate, the operational components of getting a patient from outpatient consultation to the therapeutic modality are complex. The simple timeline would undoubtably require surgical consultation, clearance for medical fitness, acquisition of operating room time and equipment, and of course the ever-present burden of approval for consultation, testing, and operation. If one simply measures the burden of an operation versus no operation with attendant potential complications, it is hard to argue or document a shorter distance from the diagnosis to first treatment when an operation is included in the neoadjuvant treatment plan. Finally, there are a very limited number of medical oncologists who appreciate a significant enough benefit of CHAI to add an operation for pump placement. After all, this is not an outpatient venous access device placement. Although, CHAI pump placement with laparoscopic or robotic technology might limit the length of stay the surgical component remains significant.

At a recent presentation of CHAI at a national oncology meeting, the therapeutic results were not challengeable with respect to disease control or theory-what was clear was the lack of awareness of the technology. Had this been something just emerging, the knowledge gap would be understandable. Unfortunately, the gap was due to the failure of the oncologic community to adopt and incorporate this therapy. Despite numerous attempts at the goal of including this in the armamentarium, there has been little success. There is no amount of discussion back and forth, persuasion, or coercion that will make a physician practice in a particular non-evidence-based manner.

One clearly appropriate aspect of the failure to adopt is that a Phase 3, randomized trial to document improvement or minimally noninferiority was never performed. The manuscript ends with the call for a multi-institutional, randomized trial. Although this suggestion is admirable, the reality is that the oncologic community, specifically those with a passion for treating resectable colorectal liver metastases, has never "gotten behind" Phase 3 trials. On a personal note, this has been one of my greatest disappointments. We need to develop an equipoise with respect to the certainties of our colleagues and test questions. The randomized, Phase 3 trial performed where the modalities are tested in compliance with strict definitions (e.g., respectable, borderline resectable, unresectable), outcome (RECIST measured response), and toxicity (CTCAE) are paramount. Sometimes I wish that the authors of papers calling for randomized trials were required to participate in said investigations - myself included. Participation would include assistance in design and most importantly insistence on accrual to the randomized options. Given the reorganization of the National Cancer Institute Cooperative Group structure, I will officially throw down the gauntletto any group-to design a trial of modern, high-response chemotherapy (e.g., FOLFOX or FOLFIRI plus cetuximab vs. CHAI with floxuridine/dexamethasone plus FOLFOX/ capecitabine) in a well-defined population of patients with borderline resectable colorectal cancer metastatic to the liver. Are there any takers?

Finally, we should be respectful of the many investigators who developed and tested CHAI. The bottom line is that the best therapies are a combination of response, toxicity, and treatment burden (time, effort, and cost). I am sure that the answer includes better cytotoxic agents, immunotherapy, and gene therapy. What is most important is to incorporate the evidence-based successes into an iterative process of continued advancement. 